\title{
CONJUGATE RADII OF QUADRIC HYPERSURFACES
}

CURTIS M. FULTON

We propose to give concise proofs of three theorems on conjugate radii of quadric hypersurfaces. Two of these theorems were shown previously $[3$, pp. 150,$160 ; 4]$. The method used there, however, seems to apply only to what one might call a hyperellipsoid. For the purpose of this paper there is no appreciable difference between the use of Riemannian coordinates in Riemannian space and Cartesian coordinates in flat space. We therefore limit our discussion to flat space referred to Cartesian coordinates $y^{i}(i=1, \cdots, n)$. Furthermore, a restriction to centro-affine transformations makes the coordinates components of a contravariant vector.

Let $a_{i j}$ be the constant components of a symmetric covariant tensor and let $e^{*}$ be plus or minus one as used in [2]. Then the equation

$$
a_{i j} y^{i} y^{j}=e^{*}
$$

represents one or two real central hyperquadrics [2, p. 202]. If two such hypersurfaces exist for given values of $a_{i j}$, they may be thought of as a generalization of conjugate hyperboloids. By definition, the points $y_{h \mid}{ }^{i}$ and $y_{k \mid}{ }^{i}$ are the extremities of two conjugate radii provided they satisfy the relations

$$
\left.a_{i j} y_{h \mid}{ }^{i} y_{k \mid}\right|^{j}=0(h \neq k), a_{i j} y_{h \mid} y_{\left.h\right|^{j}}=e_{h}^{*},\left.a_{i j} y_{k \mid}{ }^{i} y_{k \mid}\right|^{j}=e_{k}^{*},
$$

where $e_{n}^{*}$ is plus or minus one. If, as we assume, the determinant $a$ of our tensor is different from zero and $a^{i j}$ are the components of the reciprocal tensor the equations (2) are equivalent to [2, p. 96]

$$
\sum_{h} e_{h}^{*} y_{h \mid}^{i} y_{h l^{i}}=a^{i j}
$$

In what follows we will call an expression of the type occurring on the left side of (3) an algebraic sum.

Lemma. The sum $\sum_{h} e_{h}^{*}$ corresponding to $n$ conjugate radii is equal to the signature $s$ of the quadratic form $a_{i j} y^{i} y^{i}$.

Proof. We can find a system of coordinates such that $\left.y_{h \mid}\right|^{i}=\delta_{\boldsymbol{k}}^{\boldsymbol{t}}$. Then in (2) $a_{h k}=0(h \neq k)$ and $a_{h h}=e_{h}^{*}$.

THEOREM 1. The algebraic sum of the squares of $n$ conjugate radii of (1) is invariant.

Received by the editors April 25, 1960. 
Proof. Let the linear element be given by $d s^{2}=e g_{i j} d y^{i} d y^{i}[2, \mathrm{p}$. 35]. Then we have for the square of the radius pertaining to $y_{h}{ }^{i}, e_{h} s_{h}^{2}$ $=g_{i j} y_{h \mid}{ }^{i} y_{h \mid}{ }^{j}$. Hence with the aid of (3)

$$
\sum e_{h}^{*} e_{h} s_{h}^{2}=a^{i j} g_{i j} .
$$

A pair of conjugate hyperbolas illustrates a special case of this theorem.

THEOREM 2. The tangent hyperplanes at the extremities of $n$ conjugate radii of (1) meet on the quadric hypersurface $a_{i j} y^{i} y^{j}=s$.

Proof. The tangent hyperplane at $y_{h \mid}{ }^{i}$ is given by $a_{i j} y^{i} y_{h \mid}{ }^{i}=e_{h}^{*}[3$, p. 147]. Making use of (3) and the Lemma we eliminate the ends of the radii as follows

$$
s=\sum e_{h}^{*}=\sum e_{h}^{*}\left(a_{i j} y^{i} y_{\left.h\right|^{j}}\right)\left(a_{p q} y^{p} y_{h \mid}^{q}\right)=a_{i j} y^{i} a_{p q} y^{p} a^{i q}=a_{i j} y^{i} y^{j} .
$$

The latter expression is zero for conjugate hyperbolas and represents the common asymptotes.

THEOREM 3. The volume determined by $n$ conjugate radii of (1) is invariant.

Proof. If vertical bars designate determinants we infer from (2) that $\left.a\left|y_{h}\right|^{i}\right|^{2}=\left|e_{h}^{*}\right|=\operatorname{sgn} a$. On the other hand, from the usual definition of volume $[1$, p. 47],

$$
V^{2}=\left.\operatorname{sgn}(g)\left|g_{i j} y_{\left.h\right|^{i}} y_{k \mid}\right|^{i}|=\operatorname{sgn}(g) g| y_{\left.h\right|^{i}}\right|^{2}=\operatorname{sgn}(g) \operatorname{sgn}(a) g / a .
$$

\section{REFERENCES}

1. A. Duschek and W. Mayer, Lehrbuch der Differentialgeometrie, Band II, Leipzig und Berlin, Teubner, 1930.

2. L. P. Eisenhart, Riemannian geometry, Princeton, Princeton University Press, 1949.

3. C. E. Weatherburn, $A n$ introduction to Riemannian geometry, Cambridge, University Press, 1957.

4. - On certain quadric hypersurfaces in Riemannian space, Proc. Edinburgh Math. Soc. vol. 4 (1935) pp. 85-91.

University of California, Davis 